International Journal of Biomedicine | June 2021 - Volume 11, Issue Suppl_1: Abstracts from the Third Russian International Conference "Cryo-electron microscopy 2021: achievements and prospects"

POSTER ABSTRACT PRESENTATIONS

SESSION TITLE: STRUCTURE OF MEMBRANE PROTEINS

DOI: 10.21103/JJBM.11.Suppl_1.P13

\title{
Abstract P-13: Structural Studies of Insulin Receptor-Related Receptor Ectodomain
}

\author{
$\underline{\text { Andrey Mozhaev }}{ }^{1,2}$, Evgeny Pichkur ${ }^{3}$, Igor Deyev ${ }^{1}$, Ekaterina Mileshina ${ }^{1}$, \\ Anton Orekhov $^{3}$, Alexander Vasiliev ${ }^{2,3}$, Alexander Petrenko ${ }^{1}$ \\ ${ }^{1}$ Shemyakin-Ovchinnikov Institute of Bioorganic Chemistry RAS, \\ Moscow, Russia \\ ${ }^{2}$ Shubnikov Institute of Crystallography of FRSC "Crystallography and \\ Photonics" RAS, Moscow, Russia \\ ${ }^{3}$ National Research Center "Kurchatov Institute," Moscow, Russia
}

Background: The insulin receptor-related receptor (IRR) was originally discovered due to its high homology to the other family members (insulin receptor and insulin-like growth factor 1 receptor). We determined that IRR can be activated by mildly alkaline extracellular media and has typical features of the ligand-receptor interaction, including its specificity and dose-dependence. Since $\mathrm{pH}$-sensitive properties of IRR are determined by its ectodomain; therefore, we chose as an option to study the soluble extracellular domain IRR.

Methods: The investigation carried out in Titan Krios 60-300 TEM/STEM (FEI, USA) CryoEM, equipped with direct electron detector Falcon II (FEI, USA) and Cs image corrector (CEOS, Germany), at an accelerating voltage of $300 \mathrm{kV}$. Data processing and 3D reconstruction were carried out using computing resources of the Federal Collective Usage Center Complex for Simulation and Data Processing for Mega-Science Facilities at NRC "Kurchatov Institute."

Results: The obtained 2D classifications of particles of the ectodomain IRR at a neutral $\mathrm{pH}$ form several 3D models. This indicates that the ectodomain has several possible conformations, which is consistent with our previously obtained data using SEC-SAXS and AFM. In the future, additional careful data processing is required, as well as studies of the IRR ectodomain in mildly alkaline $\mathrm{pH}$. 
Conclusion: In this study, we presented the structural characteristics of the IRR ectodomain obtained by CryoEM. These results are an important step towards understanding the mechanism of functioning of the IRR.

Key Words: receptor tyrosine kinases $\bullet$ insulin receptor-related receptor •

\section{CryoEM}

This work was supported by the Russian Foundation for Basic Research (Grant No. 20-04-00959)

*Corresponding author: Andrey Mozhaev. E-mail:a.a.mozhaev@gmail.com

International Journal of Biomedicine. 2021;11 Suppl 1: S16-17.

doi: 10.21103/IJBM.11.Suppl_1.P13

(C)2021 International Medical Research and Development Corporation 